\title{
NITROGEN DOPED SUPERPOROUS CARBON PREPARED BY A MILD METHOD. ENHANCEMENT OF SUPERCAPACITOR PERFORMANCE
}

María José Mostazo-López ${ }^{1}$, Ramiro Ruiz-Rosas ${ }^{1}$, Emilia Morallón ${ }^{2, *}$, Diego Cazorla-Amorós $^{1}$

${ }^{1}$ Departamento de Química Inorgánica e Instituto Universitario de Materiales. Universidad de Alicante. Apartado 99. E-03080 Alicante. España

${ }^{2}$ Departamento de Química Física e Instituto Universitario de Materiales.

Universidad de Alicante. Apartado 99. E-03080 Alicante. España

*Corresponding author: morallon@ua.es 


\begin{abstract}
Nitrogen functionalization (ca. 4 at. $\% \mathrm{~N}_{\mathrm{XPS}}$ ) of a highly microporous activated carbon $\left(\mathrm{S}_{\mathrm{BET}}>3000 \mathrm{~m}^{2} / \mathrm{g}\right)$ has been achieved by two different approaches at mild conditions: (i) oxidation and post-reaction with nitrogen reactants, and (ii) direct reaction of pristine carbon material with nitrogen reactants. Interestingly, the introduction of nitrogen functionalities allows full preservation of the microporosity when pathway (ii) is followed. The electrochemical performance of the carbon materials as electrodes for supercapacitors was evaluated by using symmetric and asymmetric configuration in $1 \mathrm{M} \mathrm{H}_{2} \mathrm{SO}_{4}$. Both nitrogen-functionalized carbon materials showed larger stability and energy efficiency than the pristine carbon material when working at $1.4 \mathrm{~V}$. The non-oxidized and functionalized activated carbon evidences the best performance as electrode for supercapacitor, providing energy and power density of $14.5 \mathrm{Wh} / \mathrm{kg}$ and $61.2 \mathrm{~kW} / \mathrm{kg}$ and keeping $83 \%$ of original capacitance after 5000 charge-discharge cycles. This improvement is related to the presence of surface nitrogen functionalities that provide a higher electrochemical stability, avoiding the formation of detrimental oxygen groups during the operation of the supercapacitor.
\end{abstract}




\section{INTRODUCTION}

Supercapacitors have attracted considerable interest as energy storage devices thanks to their high power density, a key missing feature of fuel cells and electrical batteries. They are based on the formation of an electrical double layer on the extensive surface of porous carbon materials, which is profited to store energy that can be delivered in few seconds. This energy storage mechanism is often complemented with pseudocapacitive processes produced by fast redox reactions occurring in electroactive surface functional groups. The development of electrode materials with large surface area, electroactive functional groups and high electrochemical stability is crucial for the improvement of the energy density and durability of these devices [1,2].

Activated carbons are the most employed materials as electrodes for supercapacitors, mainly due to their large apparent surface area, proper electrical conductivity, high electrochemical stability and competitive production cost [2]. Also, their surface chemistry can be conveniently modified to introduce surface functionalities that could improve the performance of these materials by an increase of electrochemical stability, conductivity, wettability or pseudocapacitance [3]. For instance, oxygen groups and in aqueous electrolytes can increase the wettability of the surface, improving the electrolyte-electrode interaction and rendering a larger amount of the surface accessible for the formation of the electrical double layer, and can also participate in reversible faradaic reactions that contribute to the energy storage through pseudocapacitance [4]. Likewise, the presence of nitrogen on the surface of activated carbons improves the performance of activated carbon as electrodes of supercapacitors by increasing the wettability of the surface, the electrochemical stability, the conductivity or the contribution of pseudocapacitive processes [5-8]. Inversely to the ubiquity of surface oxygen groups, nitrogen functionalities are not as easily introduced in the structure of carbon materials, and the development of new procedures for obtaining nitrogencontaining porous carbons has therefore attracted a great interest.

Nitrogen-doped porous carbon materials can mainly be obtained by two pathways: reaction of the carbon material with a nitrogen-containing reagent $\left(\mathrm{NH}_{3}, \mathrm{HCN}\right.$, etc) or carbonization/activation of nitrogen-rich carbon precursors (urea, polyaniline, etc.) [6,9]. However, these approaches are usually carried out at high temperatures and, in the case of post-treatments, these conditions strongly modify the porosity of the pristine carbon material $[10,11]$. Also, the control of the nitrogen functionalities that are formed 
during post-treatments is a challenge. Therefore, post-modification treatments at low temperature are highly desirable for obtaining different nitrogen functional groups while preserving the characteristic porous texture of the original carbon material.

In our previous work [11], we proposed the modification of the surface chemistry of a highly porous activated carbon using organic chemistry reactions. Briefly, this treatment consisted in the oxidation of the carbon material in order to introduce oxygen functionalities that, afterwards, were converted into nitrogen functionalities through an amidation treatment. In a last step, the post-conversion of the formed amides into amine functional groups was achieved by a Hofmann rearrangement [12]. The modification protocol allowed the incorporation of different nitrogen functionalities. Interestingly, this method also leads to the formation of pyridines, pyridones and pyrroles, which have a positive impact in the electrochemical behavior of carbon materials $[6,8]$, but are only introduced using high temperature treatments. However, the oxidation treatment carried out during the first step of the functionalization protocol produced a remarkable decrease of the microporosity along with the generation of non-desirable functional groups ( $\mathrm{CO}_{2}$-evolving groups) from the point of view of the capacitor performance. In this work, we propose a modification of this approach that allows the introduction of nitrogen on carbon materials but preventing the previous incorporation of oxygen functional groups. The electrochemical performance of the pristine and the nitrogenfunctionalized activated carbons has been assessed as electrodes for supercapacitors.

\section{EXPERIMENTAL}

\subsection{Activated carbon}

A highly microporous activated carbon prepared in our laboratory has been used as the starting material for nitrogen incorporation via organic chemical modification. The pristine material, henceforth named KUA, has been obtained by chemical activation of a Spanish anthracite (11 wt\% of ash content) with $\mathrm{KOH}$ using an impregnation ratio of activating agent to raw material of $4: 1$ and an activation temperature of $750^{\circ} \mathrm{C}$ under inert atmosphere, which was held for 1 hour. More details about the preparation process are available elsewhere [13]. 


\subsection{Chemical functionalization of activated carbon}

\subsubsection{Synthesis of KUA-CONH2}

In our previous work [11], an approach for the incorporation of amides over activated carbon was satisfactorily developed. Also, other nitrogen functional groups were introduced on the surface of the carbon material via secondary reactions. The amidation treatment was carried out as follows:

(i) Chemical oxidation with $\mathrm{HNO}_{3}$ in order to introduce oxygen functional groups on the surface of KUA [14].

(ii) Generation of acyl chloride functionalities by reaction of the activated carbon obtained on step (i) with $\mathrm{SOCl}_{2}$ in toluene under Ar atmosphere.

(iii) Reaction of the activated carbon obtained in step (ii) with $2 \mathrm{M}$ $\mathrm{NH}_{4} \mathrm{NO}_{3} / \mathrm{DMF}$ solution and pyridine.

The obtained activated carbon was named KUA-CONH2. More details about the preparation process are available elsewhere [11].

\subsubsection{Synthesis of KUA-N}

In this case, step (iii) is directly carried out over activated carbon KUA, in order to obtain nitrogen functional groups by using a single treatment via reaction with the existing $\mathrm{CO}_{2}$ and CO-evolving groups in the pristine material. In this step (iii), 400mg of KUA were added into $140 \mathrm{~mL}$ of $2 \mathrm{M} \mathrm{NH}_{4} \mathrm{NO}_{3} / \mathrm{DMF}$ solution (activated carbon to solution ratio of $1 \mathrm{~g} / 300 \mathrm{~mL}$ ) in a round bottom flask. Then, $140 \mathrm{~mL}$ of pyridine were added slowly to the round bottom flask under continuous stirring at room temperature. The mixture was stirred at $70{ }^{\circ} \mathrm{C}$ for 65 hours. The obtained sample (KUA-N) was washed with abundant water and ethanol, filtered and dried at $100^{\circ} \mathrm{C}$ overnight.

\subsection{Porous texture and surface chemistry characterization}

The porous texture characterization was carried out by $\mathrm{N}_{2}$ adsorption-desorption isotherms at $-196^{\circ} \mathrm{C}$ and by $\mathrm{CO}_{2}$ adsorption at $0^{\circ} \mathrm{C}$ by using an Autosorb-6Quantachrome apparatus. The samples were outgassed at $200^{\circ} \mathrm{C}$ for 4 hours before the experiments. The apparent surface area was obtained from $\mathrm{N}_{2}$ adsorption isotherms by using the BET equation in the $0.05-0.20$ range of relative pressures. The total micropore volume was determined by Dubinin-Radushkevich (DR) method applied to $\mathrm{N}_{2}$ (relative 
pressures from 0.01 to 0.05 ) adsorption isotherms. The volume of the narrow microporosity (i.e., pore sizes below $0.7 \mathrm{~nm}$ ) was calculated from the DR method applied to the $\mathrm{CO}_{2}$ adsorption isotherms (relative pressures from 0.0001 to 0.25 ) [15]. The pore size distribution for both samples has been calculated from the $\mathrm{N}_{2}$ adsorption isotherms using the 2D-NLDFT Heterogeneous surface model [16] and by applying the Solution of Adsorption Integral Equation Using Splines (SAIEUS, available online at http://www.nldft.com/) Software.

The surface chemistry of the samples was analyzed by $\mathrm{X}$ Ray Photoelectron Spectroscopy (XPS) and Temperature Programmed Desorption (TPD). XPS measurements were performed by using a VG-Microtech Multilab 3000 spectrometer, equipped with an Al anode. The deconvolution of N1s spectrum was carried out by using Gaussian functions with 20\% of Lorentzian component. FWHM of the peaks was kept between 1.4 and $1.7 \mathrm{eV}$ and a Shirley line was used for estimating the background signal. TPD experiments were performed by heating the samples $(\sim 10 \mathrm{mg})$ to $950^{\circ} \mathrm{C}$ (at a heating rate of $20^{\circ} \mathrm{C} / \mathrm{min}$ ) under a helium flow rate of $100 \mathrm{~mL} / \mathrm{min}$. The analyses were carried out by using a TGA-DSC instrument (TA Instruments, SDT Q600 Simultaneous) coupled to a mass spectrometer (Thermostar, Balzers, BSC 200).

\subsection{Electrochemical characterization}

\subsubsection{Three electrode cell configuration}

Carbon electrodes for electrochemical characterization were prepared by mixing the activated carbon with acetylene black and polytetrafluoroethylene (PTFE) as binder in a ratio of 90:5:5 (w/w). The total weight of the electrode was $\sim 9 \mathrm{mg}$ (dry basis). For shaping the electrodes, a sample sheet was cut into a circular shape with an area of 1.2 $\mathrm{cm}^{2}$ and pressed for $5 \mathrm{~min}$ at 2 tons to guarantee a homogeneous thickness. After that,

the electrode was attached to a gold disk used as a current collector by using a conducting adhesive (colloidal graphite suspension, Hitasol GA-715, Hitachi Chemical Co., Ltd.). The electrodes were impregnated for 2 days into $1 \mathrm{M} \mathrm{H}_{2} \mathrm{SO}_{4}$ previously to electrochemical measurements.

The electrochemical characterization of the electrodes was performed by cyclic voltammetry in a Biologic VSP multichannel potentiostat and using a T-type 
Swagelock cell in a three-electrode configuration. $1 \mathrm{M} \mathrm{H}_{2} \mathrm{SO}_{4}$ was used as aqueous electrolyte. As counter electrode, more than $20 \mathrm{mg}$ of KUA was used. Both electrodes were tightly pressed against each other and separated by a nylon membrane (Teknokroma membrane filters, pore size: $320 \mathrm{~nm}$ ). Ag/ $\mathrm{AgCl} / \mathrm{KCl}(3 \mathrm{M})$ was used as reference electrode in all cases. The electrochemical characterization of all samples was tested by $\mathrm{CV}$ at sweep rates of 1 and $2 \mathrm{mV} / \mathrm{s}$. CV capacitance was calculated from the area of the voltammogram. The results are expressed in F/g, taking into account the weight of the active material of the working electrode.

\subsubsection{Two electrode cell configuration.}

Symmetric and asymmetric in mass capacitors were assembled for all carbon materials. For symmetric capacitors, two electrodes (surface area: $0.196 \mathrm{~cm}^{2}$ ) were prepared with a weight of $\sim 1.3 \mathrm{mg}$ (active phase) each. The thickness of the electrodes was close to 0.2 $\mathrm{mm}$ in all cases. In case of asymmetric in mass configuration, the mass of the electrodes was determined following the procedures detailed by Peng et al [17], which allows to charge the supercapacitor cells in expanded voltage with respect to the symmetric configuration, taking full profit of the stability potentials of each electrode. The electrodes were attached to a stainless steel collector by using a conducting adhesive. Supercapacitors were constructed by pressing both electrodes against each other and separating them by a nylon membrane filter (pore size: $320 \mathrm{~nm}$ ). These devices were characterized by $\mathrm{CV}$ at different scan rates, galvanostatic charge-discharge (GCD) cycles at current densities from 0.25 to $32 \mathrm{~A} / \mathrm{g}$ and Electrochemical Impedance Spectroscopy (EIS) in $1 \mathrm{M} \mathrm{H}_{2} \mathrm{SO}_{4}$ solution. Impedance spectra were measured at $0.05 \mathrm{~V}$ in the frequency range of $10 \mathrm{mHz}$ to $100 \mathrm{kHz}$ with an amplitude voltage of $10 \mathrm{mV}$. Autolab PGSTAT302 potentiostat was employed for EIS and CV measurements and Arbin SCTS potentiostat for galvanostatic charge-discharge cycles. A durability test for symmetric and asymmetric capacitors was performed by 5000 galvanostatic chargedischarge cycles at a current density of $1 \mathrm{~A} / \mathrm{g}$ and a voltage of $1.2 \mathrm{~V}$ in case of symmetric configuration and $1.4 \mathrm{~V}$ for asymmetric cells. Current density and specific capacitance is defined based on the total active weight of the carbon material included in both electrodes. 
The energy density and power density of symmetric and asymmetric supercapacitors was calculated in order to obtain all relevant information about their performance. Energy density was obtained during the discharge cycle by the following equation (1):

$\square \longdiv { 2 \square \square \square }$

Where $V_{1}$ is the cell voltage of charge $(1.2 \mathrm{~V}$ for symmetric configuration and $1.4 \mathrm{~V}$ for asymmetric configuration) and $V_{2}$ the voltage of discharge ( $0 \mathrm{~V}$ in both cases).

Power density was calculated according to equation (2):

$\square \square \square \square \square \square \square \square 24 \square \square \square \square$

Where ESR is the equivalent series resistance (determined from ohmic drop in the charge-discharge cycles), $m$ is the total active mass of the electrodes and $V_{\max }$ is the operating voltage.

\section{RESULTS AND DISCUSSION.}

\subsection{Surface chemistry and porous texture characterization.}

Table 1 shows the surface properties of the parent activated carbon and those obtained after the functionalization treatments. The results of the XPS analyses evidence that nitrogen atoms have been successfully anchored through the proposed protocols on the surface of KUA-CONH2 and KUA-N. It should be noted that both functionalization treatments have leaded to a similar nitrogen content (3.8 and 4.1 at.\% for KUA-CONH2 and KUA-N, respectively), while oxygen content increases significantly for KUACONH2 sample. The changes in surface chemistry on the sample KUA-CONH2 have been previously studied [11], and we found that this increase in oxygen was related to the chemical oxidation treatment carried out prior to amidation step. In case of KUA-N, TPD measurements show a relevant decrease in the CO-evolving groups, with a net total decrease (490 $\mu \mathrm{mol} / \mathrm{g}$ ) that is especially relevant in the region of thermal decomposition of phenol and quinone-like groups. However, XPS results evidence an increase of oxygen in both functionalized activated carbons. It should be considered that this technique only measures the atomic composition of the most external region of carbon particles, whereas TPD reflects the $\mathrm{CO}$ and $\mathrm{CO}_{2}$ evolving from the thermal decomposition of surface oxygen groups in both the inner and the outer regions of 
carbon particles. The decrease in oxygen surface groups determined by TPD indicates that part of the introduced nitrogen groups has been attached to the surface by consumption of this kind of functional groups. These reactions may lead to the formation of imines, pyridines and pyrroles [11]. The formation of these functional groups is confirmed by N1s XPS deconvolution obtained for KUA-N (Figure 1), which reveals the presence of pyrroles/pyridones $(400.4 \pm 0.2 \mathrm{eV})$, imines and pyridines (398.8 $\pm 0.2 \mathrm{eV})$ [18-21]. Also, other functionalities, such as quaternary nitrogen (401.8 \pm 0.2 $\mathrm{eV}$ ) [22], amines, amides or cyclic amides (399.6 $\pm 0.2 \mathrm{eV}$ ) [20], are formed on the surface of this carbon material. The absence of chemisorbed pyridine after both functionalization treatments was confirmed by immersing the pristine carbon in pyridine, and following this step by analogous washing and drying processes carried out for the synthesis of KUA-N and KUA-CONH2. XPS analysis revealed nitrogen content of 0.4 at. \%, that is negligible in comparison with the nitrogen content of the parent activated carbon.

Table 1. Textural properties and elemental surface composition (XPS and TPD) for the pristine and modified activated carbons.

\begin{tabular}{|c|c|c|c|c|c|c|c|}
\hline Sample & $\begin{array}{l}N_{\text {XPS }} \\
\text { (at.\%) }\end{array}$ & $\begin{array}{c}\mathbf{O}_{\mathrm{XPS}} \\
\text { (at.\%) }\end{array}$ & $\begin{array}{l}\mathrm{CO}_{2 \mathrm{TPD}} \\
(\mu \mathrm{mol} / \mathrm{g})\end{array}$ & $\begin{array}{c}C O_{\text {TPD }} \\
(\mu \mathrm{mol} / \mathrm{g})\end{array}$ & $\begin{array}{c}\mathrm{S}_{\mathrm{BET}} \\
\left(\mathrm{m}^{2} / \mathrm{g}\right)\end{array}$ & $\begin{array}{l}V_{D^{N_{2}}} \\
\left(\mathrm{~cm}^{3} / \mathrm{g}\right)\end{array}$ & $\begin{array}{l}\mathrm{V}_{\mathrm{DR} \mathrm{CO}_{2}} \\
\left(\mathrm{~cm}^{3} / \mathrm{g}\right)\end{array}$ \\
\hline KUA & 0.3 & 8.8 & 500 & 2100 & 3460 & 1.19 & 0.56 \\
\hline KUA-N & 4.1 & 11.1 & 700 & 1610 & 3450 & 1.19 & 0.55 \\
\hline KUA-CONH2 & 3.8 & 12.0 & 1030 & 2490 & 2160 & 0.74 & 0.44 \\
\hline
\end{tabular}




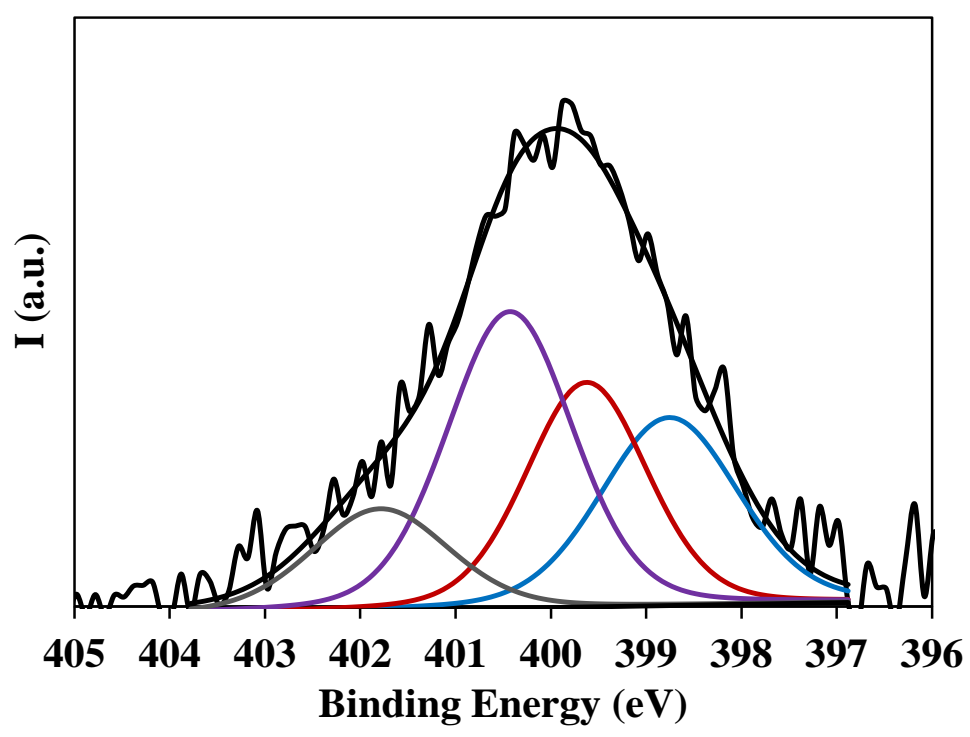

Figure 1. N1s XPS deconvolution of KUA-N.

Figure 2 shows the nitrogen adsorption-desorption isotherms of the pristine and the $\mathrm{N}$ containing activated carbons and the calculated PSD. It can be seen that the generation of functional groups on KUA-CONH2 produces a large decrease of the nitrogen uptake, which means that a noticeable part of the microporosity is blocked (Figure 2b), producing a large drop of ca. 35\% of the surface area and micropore volume, Table 1. The blockage of the porosity has been related to the damaging effect produced by the oxidation treatment on the microporosity [11]. However, when the proposed functionalization treatment is directly applied over the pristine material (obtaining KUA-N), the nitrogen adsorption isotherm superimposes with that from the pristine sample, showing that the porosity of the KUA sample does not change after the amidation treatment (KUA-N sample) due to the mild conditions used (Figure 2a). The minor differences found in the PSD (Figure 2b) are due to small discrepancies within the NL-DFT fitting of both isotherms. Thus, this pathway seems to be appropriate for functionalization of carbon materials with a high microporosity development, since it allows the incorporation of a high amount of nitrogen functionalities (4.1 at.\% XPS) along with the preservation of porous texture. 

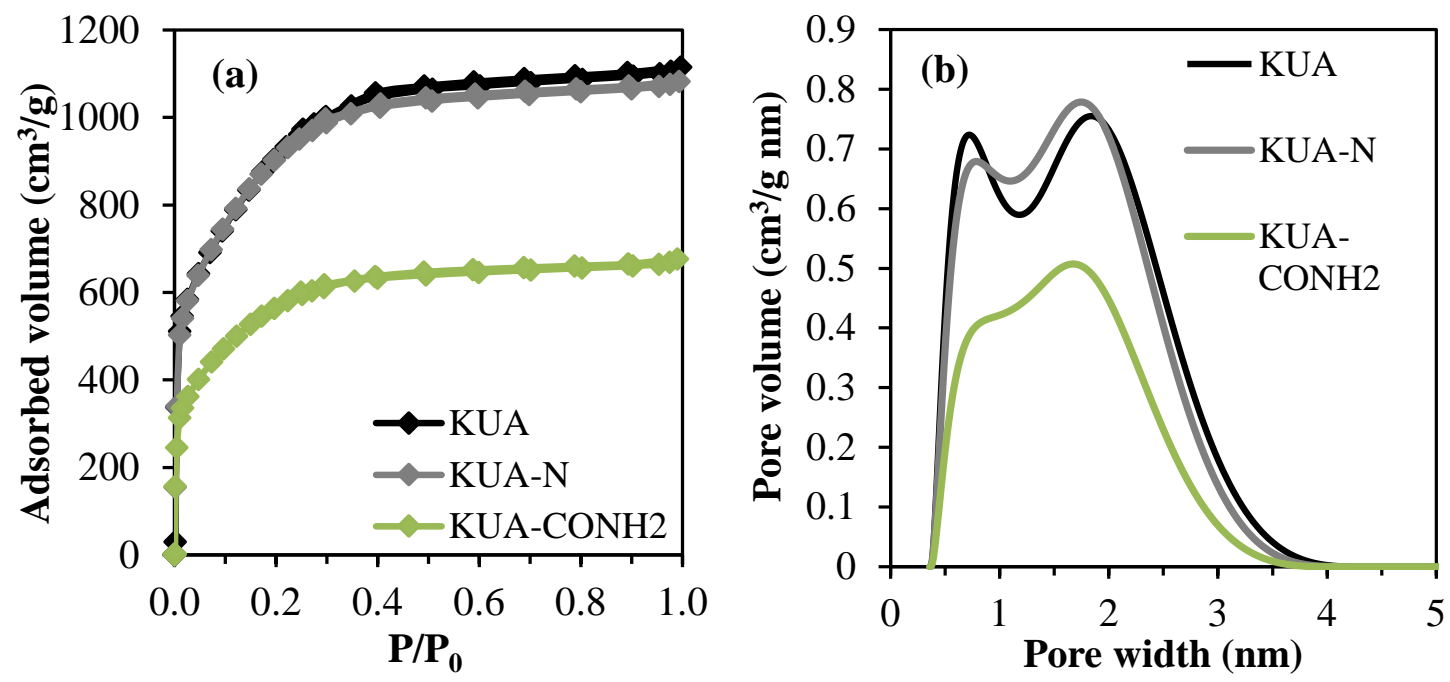

Figure 2. (a) $\mathrm{N}_{2}$ adsorption-desorption isotherms of the activated carbons KUA, KUA$\mathrm{N}$ and KUA-CONH2. (b) Pore-size distributions of KUA, KUA-N and KUA-CONH2 by DFT calculations.

\subsection{Electrochemical characterization.}

\subsubsection{Characterization of carbon materials}

The electrochemical performance of all carbon materials has been studied in a three electrode cell configuration by cyclic voltammetry. Figure 3 shows the second cyclic voltammograms for all electrodes at a potential range where they are stable (in the absence of degradation reactions). The gravimetric capacitance for all the carbon materials has been determined from CV measurements and is shown in Table 2. It can be observed that the gravimetric capacitance is mainly related to the specific area of the sample, with KUA and KUA-N showing the largest capacitance due to its largest apparent surface area, while KUA-CONH2 displays the lowest capacitance due to the decrease in porosity after the functionalization treatment.

Although sample KUA-N has a capacitance quite close to sample KUA, there are interesting differences in the shape of the voltammogram. Taking into account that this functionalization treatment does not change the apparent surface area of the pristine activated carbon, these differences are related to the changes on the surface chemistry produced by the modification protocol. Thus, KUA shows two redox processes at around $0.2 \mathrm{~V}$ and $0.4 \mathrm{~V}$, that can be related to the presence of electroactive surface 
oxygen groups [14], but KUA-N does not present these redox processes, and the electrochemical behavior shown by this electrode seems to be exclusively related to the formation of electrical double layer on the inner surface of this carbon material (Figure 3). This is in agreement with the characterization of the materials, since both have similar porous texture (Table 1 and Figure 1), but sample KUA-N has a lower content of CO-groups (Table 1) which are the electroactive oxygen functional groups in acid medium [4].

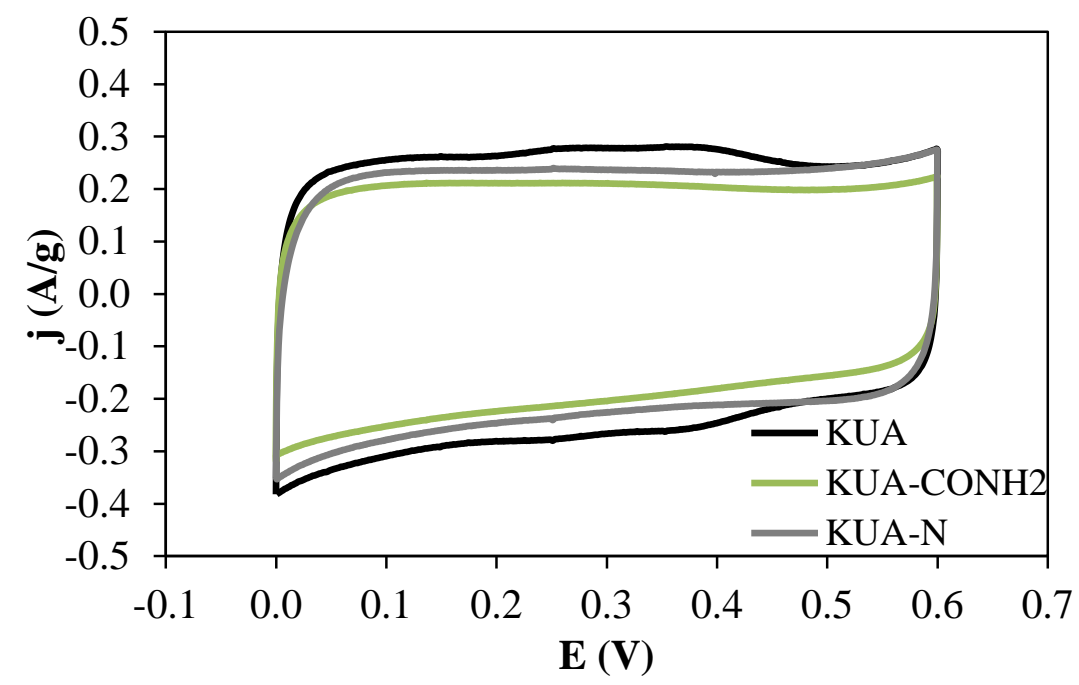

Figure 3. $2^{\text {nd }}$ cyclic voltammograms in the potential range between $0 \mathrm{~V}$ and $0.6 \mathrm{~V}$ for KUA, KUA-CONH2 and KUA-N electrodes. $1 \mathrm{M} \mathrm{H}_{2} \mathrm{SO}_{4} . \mathrm{v}=1 \mathrm{mV} / \mathrm{s}$.

Table 2. Gravimetric capacitance (Cg) determined for KUA, KUA-CONH2 and KUA$\mathrm{N}$ electrodes in the potential range between $0 \mathrm{~V}$ and $0.6 \mathrm{~V}$ by cyclic voltammetry. $1 \mathrm{M}$ $\mathrm{H}_{2} \mathrm{SO}_{4} \cdot \mathrm{v}=1 \mathrm{mV} / \mathrm{s}$.

\begin{tabular}{cc}
\hline Sample & Cg (F/g) \\
KUA & 256 \\
KUA-N & 229 \\
KUA-CONH2 & 200 \\
\hline
\end{tabular}

These changes related to the absence of electroactive oxygen functional groups in acid medium may affect the stability of the electrode. Oxygen functional groups can often be beneficial for the electrochemical performance of carbon materials since they can provide pseudocapacitance [4]; however, when these materials reach positive potentials 
$(>0.8 \mathrm{~V})$, some prejudicial functionalities, as the $\mathrm{CO}_{2}$-type groups, which are the starting point in degradation mechanism of carbon electrodes [23], can be formed. For these reasons, the potential range of stability of KUA and KUA-N have been determined in order to assess the effect of electroactive oxygen groups on the electrochemical stability of these carbon materials. Figure 4a shows the cyclic voltammograms for KUA and KUA-N in potential range between $-0.5 \mathrm{~V}$ and $0.6 \mathrm{~V}$. It is clearly seen that, beyond the differences already found in Figure 3, both electrodes present a quite similar electrochemical behavior down to $-0.2 \mathrm{~V}$. However, KUA presents a broad peak at $0.35 \mathrm{~V}$ in the negative scan that can be related to the reduction of oxygen functionalities. This peak does not appear in the case of KUA-N, evidencing the conversion of electroactive oxygen groups into different species. Figure $\mathbf{4 b}$ shows the third cyclic voltammograms for KUA and KUA-N in the potential range between $0 \mathrm{~V}$ to $1.0 \mathrm{~V}$. Again, both electrodes reveal similar performance at positive potentials evidenced by the oxidation current happening at $0.9 \mathrm{~V}$. However, the effect of the electro-oxidation on the materials is different; in the case of KUA, the redox processes at around $0.4 \mathrm{~V}$ resulting from electro-oxidation are more noticeable, mainly in the reduction one, that indicates an increase of surface oxygen groups during the positive scan, being this broad reduction peak lower in the case KUA-N activated carbon. Given that the electrochemical degradation of porous carbon materials in aqueous electrolytes seems to be related to the formation of oxidized species over the surface of the electrodes when submitted to positive potentials [24], this is a promising result in terms of a possible increase in the durability of porous carbon electrodes to positive potentials. 

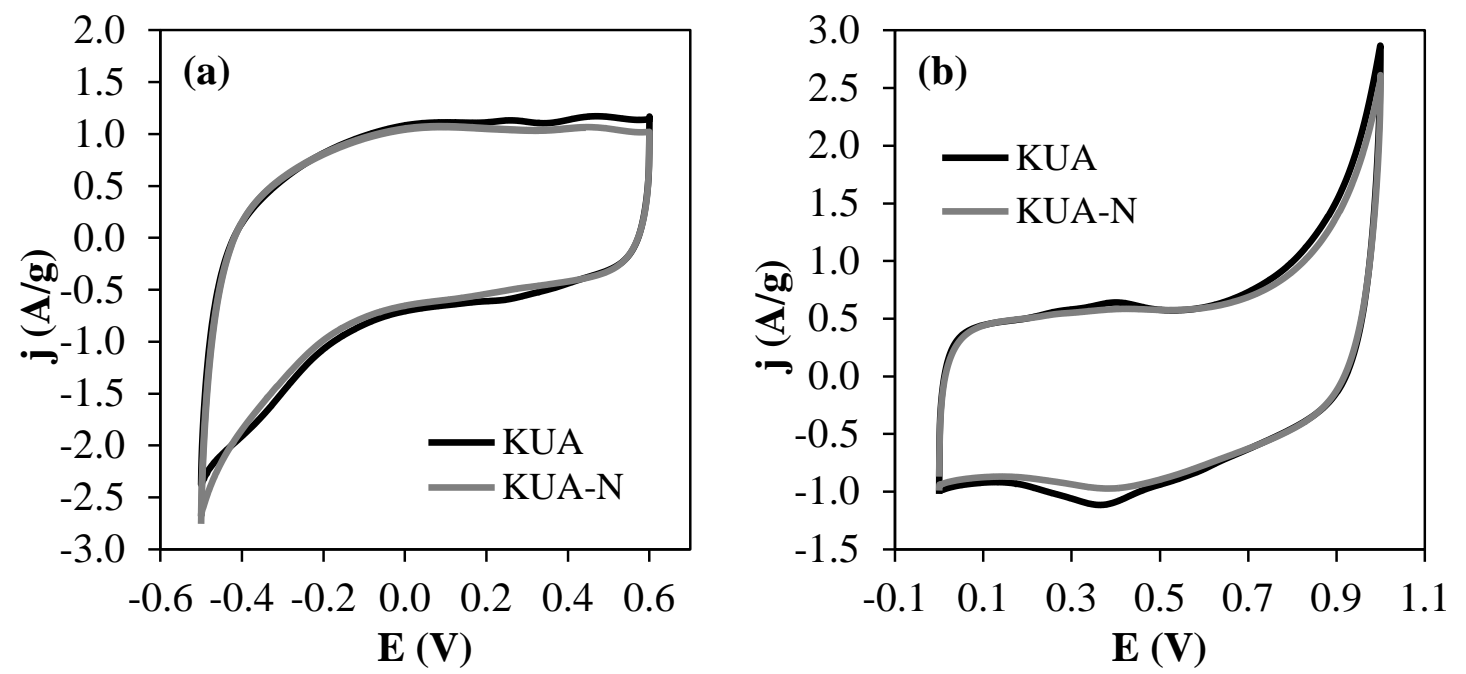

Figure 4. $3^{\text {rd }}$ cyclic voltammograms for KUA and KUA-N electrodes in the potential range (a) $-0.5-0.6 \mathrm{~V}$ and (b) $0-1.0 \mathrm{~V} .1 \mathrm{M} \mathrm{H}_{2} \mathrm{SO}_{4} . \mathrm{v}=2 \mathrm{mV} / \mathrm{s}$.

\subsubsection{Characterization of symmetric supercapacitors}

In order to study in detail the effect of surface chemistry on the performance of these materials from an application point of view, symmetric supercapacitors for the samples KUA, KUA-N and KUA-CONH2 have been studied.

Figure 5a shows the Nyquist plot obtained for all symmetric capacitors. At very high frequencies (points closer to the Y-axis), the capacitors behave like a resistance, while at low frequencies, the imaginary part of the resistance sharply increases and a curve close to a vertical line is seen, indicative of a pure capacitive behavior [25]. In the middle frequency domain, the influence of the electrode porosity and conductivity can be seen. The resistance of the cell can also be obtained from the X-axis intercept of the straight line observed at low frequencies of the diagram (the region where capacitive behavior of the capacitors is observed [26]) for all cases. The cell resistance is contributed by the resistance of charge transfer through the carbon particles and grainboundaries at the electrode-electrolyte surface (represented by a larger diameter of the semicircle with increasing the resistance [27]) and by diffusive problems of ions within the pore system (indicated by the appearance of a $45^{\circ}$ impedance line, also known as the Warburg region, after the end of the semicircle and prior to the formation of the vertical line characteristic of capacitive behavior [25]). As can be seen, KUA-CONH2 
evidences the largest resistance $(\sim 3.8 \Omega)$. This can be explained by the presence of a large amount of carboxylic acids and their cyclic species (Table 1), produced prior to the generation of nitrogen functional groups, that have an electron-withdrawing character and produce an increase of the resistance of the carbon material. The clear Warburg region observed for this sample is a consequence of the changes in porosity and the increased concentration in surface oxygen groups that hinder the mobility of ions within this pore network. KUA-N shows an even lower internal resistance than KUA. Since both samples have similar porous texture and close surface oxygen concentration (Table 1) this difference cannot be explained by diffusional problems along the porosity. Then, the improvement found for KUA-N should be related to the generation of electron-donor nitrogen functionalities [8] or to the decrease of detrimental electron-withdrawing oxygen groups improving the conductivity of the material. This positive effect of the conversion of oxygen groups into nitrogen functionalities is not observed in $\mathrm{KUA}-\mathrm{CONH}_{2}$ due to the previous generation of a large amount of carboxylic acids during the oxidation treatment with nitric acid.

Table 3. Gravimetric capacitance (Cg), energy density (E), maximum power density (P) and energy efficiency determined for KUA, KUA-CONH$H_{2}$ and KUA-N symmetric supercapacitors at the voltage $1.2 \mathrm{~V}$ by galvanostatic charge-discharge cycles. $1 \mathrm{M}$ $\mathrm{H}_{2} \mathrm{SO}_{4} \cdot \mathrm{j}=1 \mathrm{~A} / \mathrm{g}$.

\begin{tabular}{ccccc}
\hline Sample & $\begin{array}{c}\mathbf{C g} \\
\mathbf{( F / g )}\end{array}$ & $\begin{array}{c}\mathbf{E} \\
\mathbf{( W h} / \mathbf{k g})\end{array}$ & $\begin{array}{c}\mathbf{P}_{\max } \\
\mathbf{( k W / k g )}\end{array}$ & $\begin{array}{c}\text { Energy } \\
\text { efficiency } \\
\mathbf{( \% )}\end{array}$ \\
KUA & 63 & 10.3 & 43.3 & 74 \\
KUA-N & 59 & 10.5 & 72.4 & 79 \\
KUA-CONH2 & 53 & 8.7 & 20.0 & 75 \\
\hline
\end{tabular}

These differences on the resistance of the carbon material play a major role in their behavior as electrodes for supercapacitors, since the performance of supercapacitors at high power density depends on the rate of charge and discharge, which is faster when the overall resistance is lower. This explains the best performance observed for KUA-N in the Ragone plot (measured using GCD experiments at $1.2 \mathrm{~V}$ under different specific currents) shown in Figure 5b. This supercapacitor keeps larger energy density at high 
power density due to its lower resistance, and provides a maximum power density of $72.4 \mathrm{~kW} / \mathrm{kg}$ (Table 3). As expected, KUA shows similar energy density and capacitance at low power density $(10.3 \mathrm{Wh} / \mathrm{kg}$ for KUA and $10.5 \mathrm{Wh} / \mathrm{kg}$ for KUA-N at $0.5 \mathrm{~kW} / \mathrm{kg}$, and gravimetric capacitances of $63 \mathrm{~F} / \mathrm{g}$ vs $59 \mathrm{~F} / \mathrm{g}$, respectively) but is not able to keep these values when power demand is high due to its larger internal resistance. This is confirmed in Figure 5c and Figure 5d, where galvanostatic charge-discharge profiles are shown at different current densities. As can be observed in Figure 5c, at $1 \mathrm{~A} / \mathrm{g}$, KUA and KUA-N based symmetric capacitors show similar capacitance (Table 3) (which is derived from the discharge time of the profiles) due to its similar apparent surface area as was pointed out in section 3.2.1. Although KUA shows a slightly higher capacitance, KUA-N shows better performance as supercapacitor due to the lower presence of electroactive species, which make more efficient the charge-discharge process. This can be noticed in the shape of the profile, which is closer to the ideal triangular shape in the case of KUA-N, and also in the energy efficiency (i.e. the ratio between the energy employed in charging the cell and the energy recovered upon discharge of the cell) of the KUA-N capacitor (Table 3). Also, at high current density (16 A/g), Figure 5d, the best performance of KUA-N is pointed out by the lower ohmic drop produced during the transition of charge to discharge in the GCD profile, which provides a larger capacitance retention at high current. KUA-CONH2 based capacitor evidences lower capacitance and energy density than the other supercapacitors as consequence of its lower porosity. Also, its maximum power density is the lowest (Table 3) due to the detrimental effect of $\mathrm{CO}_{2}$-evolving groups on the resistance of the carbon material (Figure 1a) that produces a larger ohmic drop and, consequently, a loss of power density. 

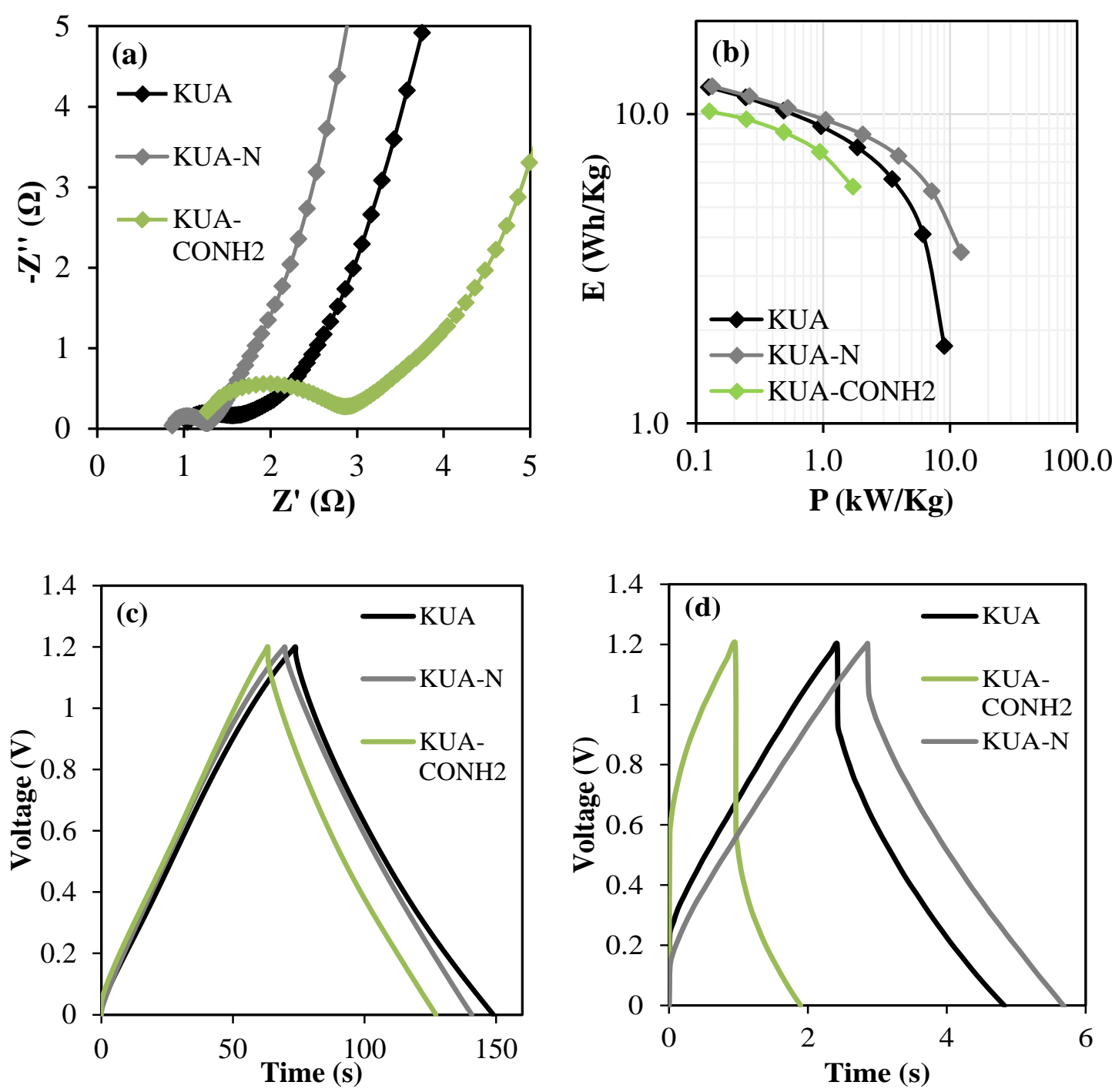

Figure 5. (a) Nyquist plot for the symmetric supercapacitors KUA, KUA-N and KUACONH2. (b) Ragone plot at $1.2 \mathrm{~V}$ for all symmetric supercapacitors. Galvanostatic charge-discharge cycles for the samples at (c) $1 \mathrm{~A} / \mathrm{g}$ and (d) $16 \mathrm{~A} / \mathrm{g} .1 \mathrm{M} \mathrm{H}_{2} \mathrm{SO}_{4}$ solution.

The effect of nitrogen functionalities upon the durability of these capacitors has been assessed by carrying out 5000 cycles of galvanostatic charge-discharge at $1 \mathrm{~A} / \mathrm{g}$ and $1.2 \mathrm{~V}$ of cut-off voltage. Figure 6 shows the evolution of gravimetric capacitance along the whole experiment. All the capacitor cells behaved adequately upon the test, showing a capacitance retention higher than 92\%. The better electrochemical stability of a nitrogen-containing activated carbon obtained by carbonization of PANI-functionalized KUA in organic media has been recently demonstrated [6], and seems to be also found 
in aqueous electrolyte. Given the possibility of achieving a larger stability in these materials, asymmetric in mass capacitors have been constructed from KUA and KUA-N electrodes.

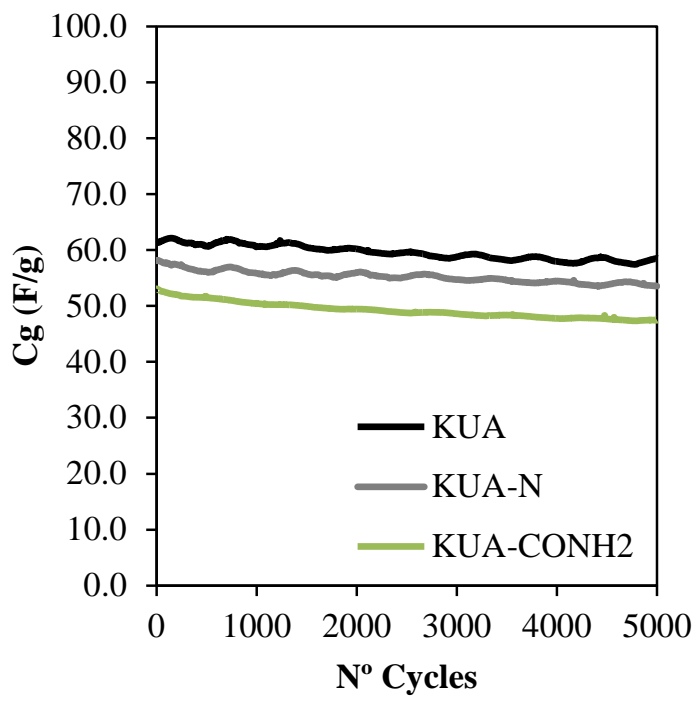

Figure 6. Cyclability test for KUA, KUA-N and KUA-CONH2 supercapacitors at 1.2V. 1A/g. 5000 cycles. $1 \mathrm{M} \mathrm{H}_{2} \mathrm{SO}_{4}$ solution.

\subsubsection{Characterization of asymmetric supercapacitors}

Asymmetric in mass supercapacitors, where the cells are set up with the same carbon material as positive and negative electrode, but with different weights, have been obtained using the method proposed by Peng et al. [17]. Briefly, this method consists in $\Delta \square_{+}$and $\Delta \square$ ) of electrochemical stability for the positive and negative electrodes from the open circuit potential (E $\square+\square$ ) of the electrodes by equaling the charge stored in each electrode while fixing the values of the potential windows to those determined in (i). The expression used in the last step reads as follows:

$\square+\square=\square \square(-\mid \Delta \square-1(\square \square+\cdot \Delta \square+)$

Then, by using the capacitance of each electrode in Eqn. 3, the mass ratio for the asymmetric supercapacitors was calculated to be 1.31, 1.12 and 1.02 for KUA, KUA-N and KUA-CONH2, respectively. Table 4 shows the capacitances and potential window values, the weight of the electrodes employed for the construction of each capacitor cell 
and the open circuit potential of each carbon material. It is important to note that the sum of the potential windows of each electrode corresponds to the working voltage of the capacitor, and that the three cells will be able to operate up to $1.4 \mathrm{~V}$. Such a high voltage is expected to increase the energy density of the capacitors, but will also expose the capacitor to harsh conditions, where the possible influence of nitrogen functionalities could be observed.

Table 4. Parameters employed for the design of asymmetric capacitors, and open circuit potential for each carbon material. $1 \mathrm{M} \mathrm{H}_{2} \mathrm{SO}_{4}$.

\begin{tabular}{|c|c|c|c|c|c|c|c|}
\hline Supercapacitor & $\begin{array}{l}D \| D_{+} \\
\mathbf{( F / g )}\end{array}$ & $\begin{array}{l}U \mathbb{Z} \\
\text { (F/g) }\end{array}$ & $\Delta[\bar{\Delta} \mathbf{( V )}$ & $\begin{array}{l}\Delta L \\
\text { (V) }\end{array}$ & $\begin{array}{c}t+ \\
\text { (mg) }\end{array}$ & $\begin{array}{c}\square f \\
\text { (mg) }\end{array}$ & $\begin{array}{c}E_{O C P} \\
(V)\end{array}$ \\
\hline KUA & 263 & 298 & 0.65 & 0.75 & 1.30 & 1.05 & 0.25 \\
\hline KUA-N & 252 & 283 & 0.70 & 0.70 & 1.22 & 1.08 & 0.20 \\
\hline $\mathrm{KUA-CONH}_{2}$ & 213 & 199 & 0.67 & 0.73 & 1.18 & 1.14 & 0.23 \\
\hline
\end{tabular}

Figure 7a shows galvanostatic charge-discharge cycles obtained for the asymmetric supercapacitors at $1 \mathrm{~A} / \mathrm{g}$. In this case, the capacitance obtained for all asymmetric supercapacitors follows the same order as for the symmetric capacitors: KUA > KUA$\mathrm{N}>>$ KUA-CONH2. However, KUA-N based capacitor provides the lower ohmic drop due to the lower electrical resistance of this carbon material, which provides the highest power density. Again, a quasi-triangular shape profile is observed in the case of KUA-N based capacitor. Figure 7b shows the Ragone plot obtained for all asymmetric supercapacitors working at $1.4 \mathrm{~V}$. As in the case of symmetric devices (section 3.2.2), KUA and KUA-N based capacitors evidence largest energy density than KUA-CONH2 (Table 5) due to their larger apparent surface area. The huge potential of the asymmetric in mass design for enhancing energy storage can be observed when the energy densities achieved in this configuration and those obtained in the symmetric design are compared (Table 3). It is important to note that KUA-N based capacitor shows a slightly higher energy density than KUA in spite of having lower capacitance, what indicates again the better performance of this material as electrode for supercapacitor. In addition, KUA-N based asymmetric capacitor is able to maintain the superior power density that has already shown in symmetric configuration. 

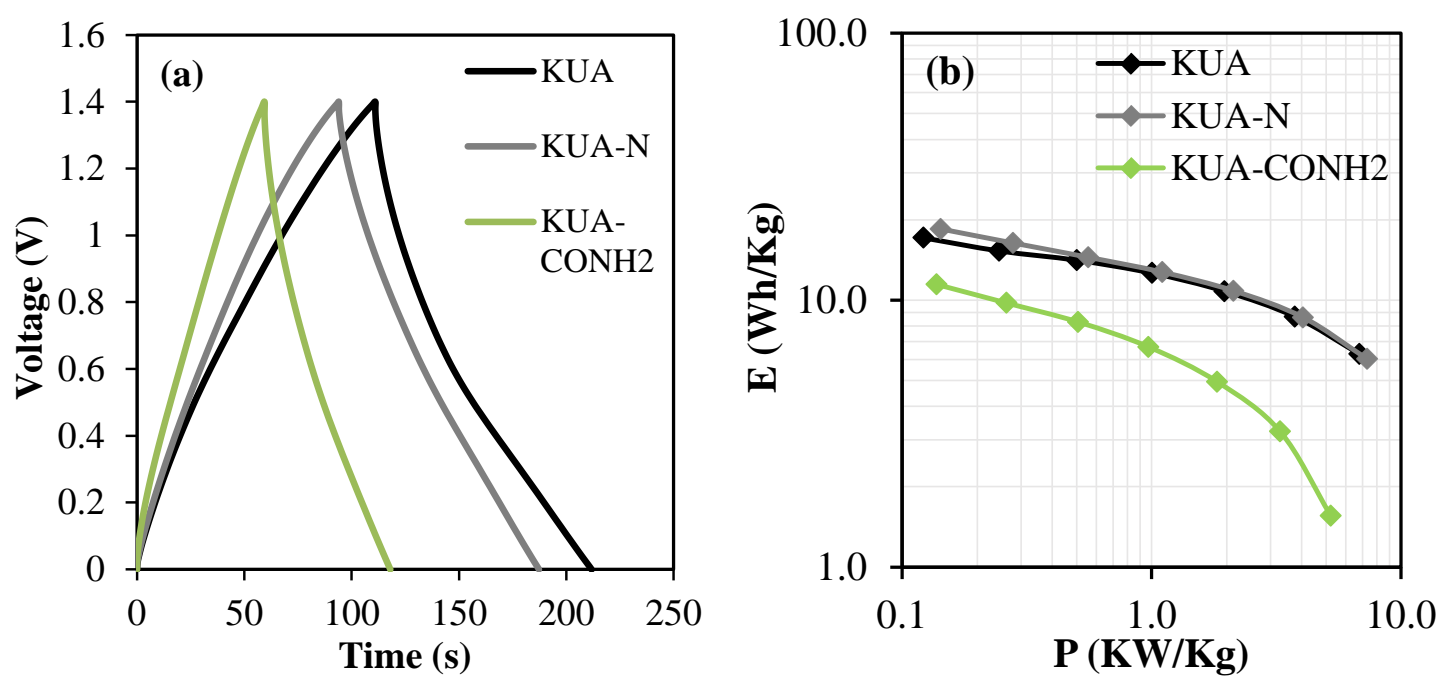

Figure 7. (a) Galvanostatic charge-discharge cycles for KUA, KUA-N and KUACONH2 asymmetric supercapacitors at $1.4 \mathrm{~V}$ and $1 \mathrm{~A} / \mathrm{g}$. (b) Ragone plot at $1.4 \mathrm{~V}$ for all asymmetric supercapacitors.

Table 5. Gravimetric capacitance (Cg), energy density (E), power density (P), coulombic efficiency and energy efficiency determined for KUA, KUA-CONH2 and KUA-N asymmetric supercapacitors at the voltage $1.4 \mathrm{~V}$ by galvanostatic chargedischarge cycles. $1 \mathrm{M} \mathrm{H}_{2} \mathrm{SO}_{4}$. j=1A/g.

\begin{tabular}{cccccc}
\hline Sample & $\mathbf{C g}$ & $\mathbf{E}$ & $\mathbf{P}_{\max }$ & $\begin{array}{c}\text { Coulombic } \\
\text { efficiency (\%) }\end{array}$ & $\begin{array}{c}\text { Energy } \\
\text { efficiency (\%) }\end{array}$ \\
KUA & 72 & 14.1 & 52.5 & 90 & 55 \\
KUA-N & 67 & 14.5 & 61.2 & 99 & 66 \\
KUA-CONH & 41 & 8.3 & 31.6 & 98 & 62 \\
\hline
\end{tabular}

The most remarkable result is the improvement in coulombic and energy efficiency observed in the case of those supercapacitors constructed with nitrogen-functionalized activated carbon as electrodes (KUA-N and KUA-CONH2), Table 5. Coulombic efficiency is under the allowable limit in the case of KUA, and points out that either massive electrolyte decomposition or carbon degradation is occurring in this capacitor. On the other hand, the coulombic efficiency is close to $100 \%$ in the case of the $\mathrm{N}$ containing carbon capacitors. This fact clearly evidences that the presence on the surface of nitrogen functional groups can avoid the irreversible faradaic reactions that occur at the surface of carbon materials in the positive electrode and that are connected 
to the malfunctioning of aqueous capacitor cells. More striking results can be seen on the durability test conducted over these capacitors, Figure 8a. The profile of capacitance for KUA-N and KUA-CONH2 based capacitors is similar during the GCD test at $1 \mathrm{~A} / \mathrm{g}$ for 5000 cycles, and when compared to that of the corresponding symmetric capacitors (Figure 6), a slightly higher capacitance drop can be seen in this case, providing a capacitance retention of 83 and 85\%, respectively. This is an expected result that can be explained by the higher cut-off voltage of these capacitors, which forces both electrodes to work closer to its electrochemical stability limits. On the other hand, the performance of the cell assembled using KUA shows some oscillations during the duration of the test, rendering unreliable the KUA asymmetric capacitor. This behavior is probably connected to the evolution of gases in this cell during the test. The absence of such problems in the capacitors constructed using $\mathrm{N}$-containing carbons undoubtedly speaks about the impact of nitrogen groups upon the electrochemical stability of activated carbons in aqueous media. However, after around 3000 cycles, the capacitance stabilizes.

Ragone plot of asymmetric capacitors was measured again after durability test, Figure 8b. These results evidence that KUA-N and KUA-CONH2 based asymmetric supercapacitors keep most of their original performance, since they provide high energy densities at high power density. However, KUA based asymmetric capacitor shows an overall decrease of energy density, which is especially large at high power density. It should be noted that, during the last 2000 cycles of durability test, the gravimetric capacitance determined for KUA is slightly larger than that of KUA-N. However, KUA$\mathrm{N}$ provides higher energy density (Figure $8 \mathrm{~b}$ ) evidencing again the importance of the energy efficiency achieved during the charge-discharge processes. The generation of detrimental functional groups on KUA during cycles along with irreversible reactions occurring on the electrodes in this supercapacitor decreases coulombic and energy efficiencies, explaining why KUA has a lower energy density than KUA-N even when its gravimetric capacitance is higher. Also, a decrease of maximum power of $62 \%$ is attained for KUA capacitor, which is a much severe drop than those obtained for KUA$\mathrm{N}$ and KUA-CONH2 based asymmetric capacitors (37 and 14\%, respectively). This loss of power density confirms the improved stability of nitrogen-containing activated carbons as electrodes for asymmetric supercapacitors in aqueous electrolyte. 

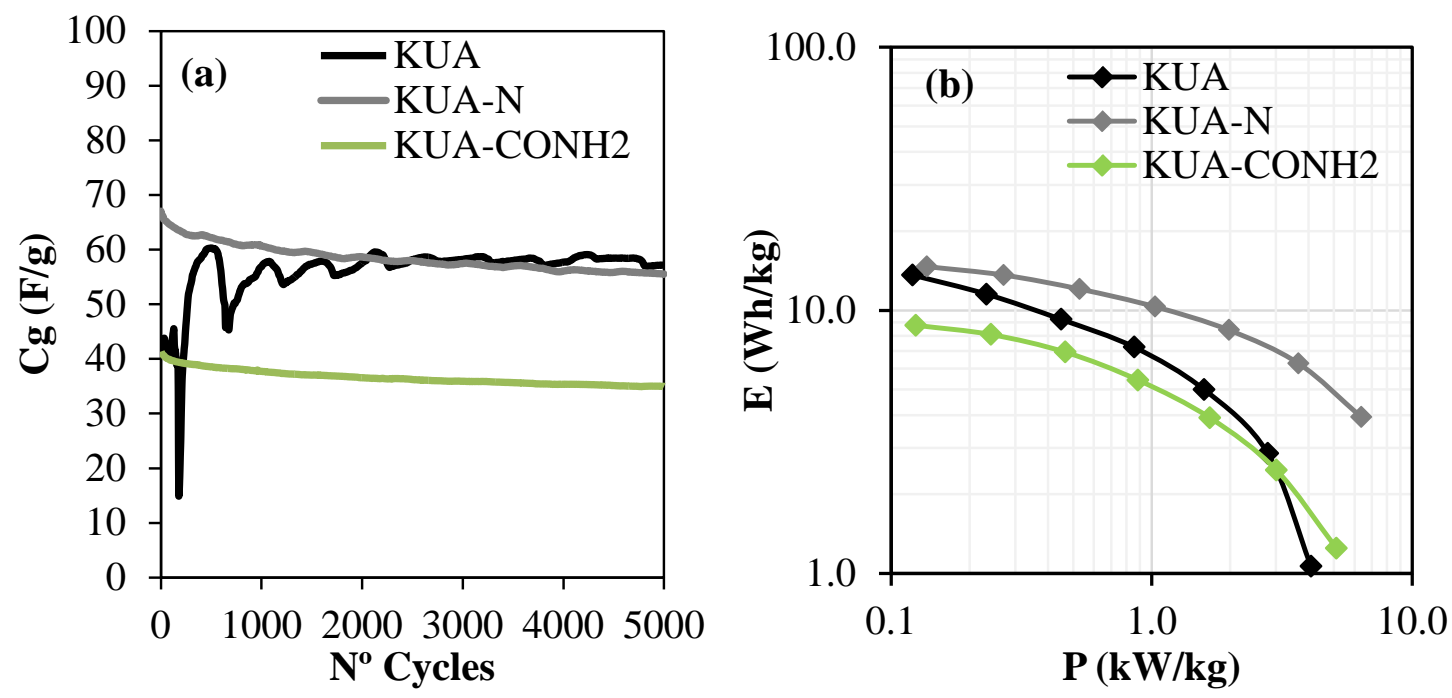

Figure 8. (a) Durability test for KUA, KUA-N and KUA-CONH2 based asymmetric capacitors at $1.4 \mathrm{~V}$, specific current of $1 \mathrm{~A} / \mathrm{g}$ and 5000 cycles. (b) Ragone plot obtained after durability test for KUA, KUA-N and KUA-CONH2 based supercapacitors.

Table 6. Energy density (E), power density (P), and energy efficiency determined for KUA, KUA-CONH2 and KUA-N asymmetric supercapacitors after durability test at the voltage $1.4 \mathrm{~V}$ by galvanostatic charge-discharge cycles. $1 \mathrm{M} \mathrm{H}_{2} \mathrm{SO}_{4} . \mathrm{j}=4 \mathrm{~A} / \mathrm{g}$.

\begin{tabular}{|c|c|c|c|c|c|}
\hline Sample & $\begin{array}{c}\mathrm{Cg} \\
(\mathrm{F} / \mathrm{g})\end{array}$ & $\begin{array}{c}E \\
(W h / k g)\end{array}$ & $\begin{array}{c}\mathbf{P}_{\max } \\
(\mathrm{kW} / \mathrm{kg})\end{array}$ & $\begin{array}{c}\text { Energy } \\
\text { efficiency (\%) }\end{array}$ & $\begin{array}{c}\text { Coulombic } \\
\text { efficiency (\%) }\end{array}$ \\
\hline KUA & 32.2 & 5.0 & 19.7 & 45 & 98 \\
\hline KUA-N & 44.3 & 8.4 & 38.9 & 60 & 99 \\
\hline KUA-CONH2 & 23.9 & 3.9 & 27.1 & 49 & 98 \\
\hline
\end{tabular}

\section{Conclusions}

Chemical functionalization of an activated carbon with a high surface area was carried out by following two different pathways: (i) amidation of an activated carbon previously oxidized (KUA-CONH2) and (ii) direct treatment of the pristine activated carbon with the same nitrogen reagents (KUA-N). Both treatments lead to a similar content of nitrogen species ( $\sim 4$ at. $\%$ XPS). The amidation treatment for generation of KUA-CONH2 produces a decrease of $30 \%$ of microporosity due to the detrimental effect of the oxidation step. In case of KUA-N, this step is not carried out and consequently the obtained carbon material preserves $100 \%$ of the porous texture of the 
pristine carbon material, making this single-step treatment more appropriate for functionalization of highly microporous carbon materials, which is applied at mild conditions.

The surface chemistry and porous texture of these carbon materials clearly influence the electrochemical performance of these carbon materials. KUA-CONH2 showed the lower capacitance due to its decrease in porosity. In addition, KUA-N evidences a slight decrease of capacitance due to the removal of electroactive oxygen groups. On the other hand, impedance spectroscopy showed that the presence of nitrogen functionalities in KUA-N promoted the conductivity of the electrode, facilitate ion diffusion and electrolyte polarization. The effect of these changes on the electrochemical performance of these carbon materials was thoroughly analyzed by studying their performance as electrodes of symmetric and asymmetric supercapacitors. KUA-CONH2 based capacitors showed the lower energy density along with a lower power density as consequence of the presence of a larger amount of $\mathrm{CO}_{2}$-evolving groups, which strongly affect the conductivity of the carbon material and the whole device. However, KUA-N based capacitors showed similar energy density, better capacitance retention and higher power density than the pristine activated carbon as consequence of the beneficial effect of the generation of electron-donor nitrogen groups and the removal of detrimental electroactive oxygen functionalities, producing a supercapacitor with higher energy and coulombic efficiency. Also, both nitrogen-functionalized activated carbons revealed higher stability than the pristine activated carbon, which is not able to safely operate at $1.4 \mathrm{~V}$ voltage in aqueous medium. Since no changes have been made in the textural properties of the parent carbon, the improvements found for KUA-N based capacitors are undoubtedly related to the presence of stable nitrogen functionalities in the activated carbon.

\section{Acknowledgements}

This work was supported by the Ministry of Economy and competitiveness of Spain (MINECO) and FEDER (CTQ2015-66080-R, MAT2013-42007-P) and Generalitat Valenciana (PROMETEO/2013/038 and PROMETEOII/2014/010). RRR thanks MINECO for 'Juan de la Cierva' contract (JCI-2012-12664). MJML acknowledges Generalitat Valenciana for the financial support through a VALi+d contract (ACIF/2015/374). 


\section{References}

[1] Simon P, Gogotsi Y. Materials for electrochemical capacitors. Nat Mater 2008;7:845-54. doi:10.1038/nmat2297.

[2] Béguin F, Frackowiak E, editors. Carbons for Electrochemical Energy Storage and Conversion Systems. 1st ed. CRC Press; 2009.

[3] Bandosz TJ, Ania CO. Surface chemistry of activated carbons and its characterization. In: Bandosz TJ, editor. Act. carbon surfaces Environ. Remediat. 1st ed., Elsevier; 2006, p. 159-229.

[4] Bleda-Martínez MJ, Maciá-Agulló JA, Lozano-Castelló D, Morallón E, CazorlaAmorós D, Linares-Solano A. Role of surface chemistry on electric double layer capacitance of carbon materials. Carbon 2005;43:2677-84.

doi:10.1016/j.carbon.2005.05.027.

[5] Hulicova-Jurcakova D, Kodama M, Shiraishi S, Hatori H, Zhu ZH, Lu GQ. Nitrogen-Enriched Nonporous Carbon Electrodes with Extraordinary Supercapacitance. Adv Funct Mater 2009;19:1800-9. doi:10.1002/adfm.200801100.

[6] Salinas-Torres D, Shiraishi S, Morallón E, Cazorla-Amorós D. Improvement of carbon materials performance by nitrogen functional groups in electrochemical capacitors in organic electrolyte at severe conditions. Carbon 2015;82:205-13. doi:10.1016/j.carbon.2014.10.064.

[7] Hsieh C-T, Teng H, Chen W-Y, Cheng Y-S. Synthesis, characterization, and electrochemical capacitance of amino-functionalized carbon nanotube/carbon paper electrodes. Carbon 2010;48:4219-29. doi:10.1016/j.carbon.2010.07.021.

[8] Ornelas O, Sieben JM, Ruiz-Rosas R, Morallón E, Cazorla-Amorós D, Geng J, et al. On the origin of the high capacitance of nitrogen-containing carbon nanotubes in acidic and alkaline electrolytes. Chem Commun 2014;50:11343-6. doi:10.1039/c4cc04876h.

[9] Shen W, Fan W. Nitrogen-containing porous carbons: synthesis and application. J Mater Chem A 2013;1:999. doi:10.1039/c2ta00028h. 
[10] Tamai H, Shiraki K, Shiono T, Yasuda H. Surface functionalization of mesoporous and microporous activated carbons by immobilization of diamine. J Colloid Interface Sci 2006;295:299-302. doi:10.1016/j.jcis.2005.08.012.

[11] Mostazo-López MJ, Ruiz-Rosas R, Morallón E, Cazorla-Amorós D. Generation of nitrogen functionalities on activated carbons by amidation reactions and Hofmann rearrangement: Chemical and electrochemical characterization. Carbon 2015;91:252-65. doi:10.1016/j.carbon.2015.04.089.

[12] Gromov A, Dittmer S, Svensson J, Nerushev OA, Perez-García SA, LiceaJiménez L, et al. Covalent amino-functionalisation of single-wall carbon nanotubes. J Mater Chem 2005;15:3334-9. doi:10.1039/b504282h.

[13] Lozano-Castelló D, Lillo-Ródenas MA, Cazorla-Amorós D, Linares-Solano A. Preparation of activated carbons from Spanish anthracite. Carbon 2001;39:741-9. doi:10.1016/S0008-6223(00)00185-8.

[14] Bleda-Martínez MJ, Lozano-Castelló D, Morallón E, Cazorla-Amorós D, Linares-Solano A. Chemical and electrochemical characterization of porous carbon materials. Carbon 2006;44:2642-51. doi:10.1016/j.carbon.2006.04.017.

[15] Cazorla-Amorós D, Alcañiz-Monge J, De La Casa-Lillo MA, Linares-Solano A. CO2 as an adsorptive to characterize carbon molecular sieves and activated carbons. Langmuir 1998;14:4589-96. doi: 10.1021/la980198p

[16] Jagiello J, Olivier JP. 2D-NLDFT adsorption models for carbon slit-shaped pores with surface energetical heterogeneity and geometrical corrugation. Carbon 2013;55:70-80. doi:10.1016/j.carbon.2012.12.011.

[17] Peng C, Zhang S, Zhou X, Chen GZ. Unequalisation of electrode capacitances for enhanced energy capacity in asymmetrical supercapacitors. Energy Environ Sci 2010;3:1499. doi:10.1039/c0ee00228c.

[18] Raymundo-Piñero E, Cazorla-Amorós D, Linares-Solano A. The role of different nitrogen functional groups on the removal of $\mathrm{SO} 2$ from flue gases by $\mathrm{N}$ doped activated carbon powders and fibres. Carbon 2003;41:1925-32. doi:10.1016/S0008-6223(03)00180-5. 
[19] Raymundo-Piñero E, Cazorla-Amorós D, Linares-Solano A, Find J, Wild U, Schlögl R. Structural characterization of N-containing activated carbon fibers prepared from a low softening point petroleum pitch and a melamine resin. Carbon 2002;40:597-608. doi:10.1016/S0008-6223(01)00155-5.

[20] Jansen RJJ, van Bekkum H. XPS of nitrogen-containing functional groups on activated carbon. Carbon 1995;33:1021-7. doi:10.1016/0008-6223(95)00030-H.

[21] Yamada Y, Kim J, Matsuo S, Sato S. Nitrogen-containing graphene analyzed by X-ray photoelectron spectroscopy. Carbon 2014;70:59-74. doi:10.1016/j.carbon.2013.12.061.

[22] Kapteijn F, Moulijn J a., Matzner S, Boehm H-P. The development of nitrogen functionality in model chars during gasification in $\mathrm{CO} 2$ and O2. Carbon 1999;37:1143-50. doi:10.1016/S0008-6223(98)00312-1.

[23] Berenguer R, Ruiz-Rosas R, Gallardo A, Cazorla-Amorós D, Morallón E, Nishihara H, et al. Enhanced electro-oxidation resistance of carbon electrodes induced by phosphorus surface groups. Carbon 2015;95:681-9. doi:10.1016/j.carbon.2015.08.101.

[24] Su-II P, Eung-Jo L, Tae-Young K, Seo-Jae L, Young-Gyoon R, Chang-Soo K. Role of surface oxides in corrosion of carbon black in phosphoric acid solution at elevated temperature. Carbon 1994;32:155-9. doi:10.1016/0008-6223(94)900205.

[25] Kötz R, Carlen M. Principles and applications of electrochemical capacitors. Electrochim Acta 2000;45:2483-98.

[26] Conway BE. Electrochemical supercapacitors: Scientific Fundamentals and Technological Applications. New York: Springer; 1999.

[27] Fletcher S, Black VJ, Kirkpatrick I. A universal equivalent circuit for carbonbased supercapacitors. J Solid State Electrochem 2014;18:1377-87. doi:10.1007/s10008-013-2328-4. 